\title{
Immune Complex Mediated Membranoproliferative Glomerulonephritis
}

National Cancer Institute

\section{Source}

National Cancer Institute. Immune Complex Mediated Membranoproliferative

Glomerulonephritis. NCl Thesaurus. Code C123055.

Glomerulonephritis characterized by mesangial proliferation, endocapillary proliferation, and glomerular capillary wall remodeling with immune complex deposits from classical complement pathway activation. 